\title{
Parasite immunomodulation and polymorphisms of the immune system
}

\author{
Rick M Maizels
}

Address: Centre for Immunity, Infection and Evolution, and Institute of Immunology and Infection Research, University of Edinburgh, Edinburgh, EH9 3JT, UK. Email: rick.maizels@ed.ac.uk

\begin{abstract}
Parasites are accomplished evaders of host immunity. Their evasion strategies have shaped every facet of the immune system, driving diversity within gene families and immune gene polymorphisms within populations. New studies published recently in BMC Biology and Journal of Experimental Medicine document parasite-associated immunosuppression in natural populations and suggest that host genetic variants favoring resistance to parasites may be detrimental in the absence of infection.
\end{abstract}

\section{Parasites}

Parasites are eukaryotic pathogens, and broadly comprise protozoa, fungi, helminths and arthropods (Figure 1) that complete part or all of their life cycle within a host organism. Like other pathogens, parasites must survive in the face of a highly potent immune system. They succeed in this through a great diversity of strategies for avoiding immune detection, suppressing cellular immunity and deflecting immune attack mechanisms. It has been suggested that the need to overcome suppressive mechanisms of parasites may have led to compensatory adjustments in immune genes that, in an environment where parasitic infection is not endemic, may increase the likelihood of inappropriate responsiveness to self-antigens (autoimmunity) and environmental allergens (allergy). This notion has become known as the hygiene hypothesis [1]. Two recent papers, from Jackson et al. [2] and Fumagalli et al. [3] lend support to this hypothesis.

\section{The immune response to parasitic infection}

The first line of defense against parasites, as with other pathogens, is the innate immune system, which is 'hardwired' (faithful to genomic sequence) and primed even in the absence of infection. It is characterized by families of molecules - serum proteins and intracellular and cellsurface receptors - known as pattern recognition receptors (PRRs) that recognize generic molecular structures associated with different groups of pathogens. Among other actions, these receptors mobilize macrophages and granulocytes, unleashing antimicrobial proteins and reactive metabolites. They also mobilize dendritic cells, which activate the lymphocytes of the adaptive immune system, inducing proliferation of $\mathrm{T}$ cells and antibodyproducing $\mathrm{B}$ cells with variable receptors that specifically recognize the parasite.

The canonical pattern-recognition receptor of the innate immune system is the cell surface Toll-Like Receptor-4 (TLR-4), which binds to the cell wall lipopolysaccharide (LPS) of Gram-negative bacteria [4]. Detailed phylogenetic analysis of the TLR family indicates strong conservation of sequence and function [5], but there is significant finedetail polymorphism across the TLR-related pathway within the human population, linked to differences in immune responsiveness to bacterial infection [4]. Why would such polymorphisms exist? It is most likely that they are maintained by variations in TLR ligands among pathogens. But while the function of the innate receptors is to activate immediate reactions to microbial infection, some eukaryotic parasites can negatively signal through the same receptors [6], suggesting a complex trade-off for the host, resulting in selection of both ligand-binding and signaling variants which would resist pathogen repression.

Innate immunity alone seldom eliminates successful parasites, but it inhibits growth while recruiting the antigen-specific $\mathrm{T}$ and $\mathrm{B}$ cells of the adaptive immune system to proliferate and differentiate into effector cells competent to attack the infection. It is therefore the evasion of adaptive immunity that is indispensable to parasite survival [7], and for rapidly proliferating protozoa an effective evasion strategy is antigenic variation, in which the expression of distinct surface molecules allows new variants to escape immune recognition, quickly replacing those killed by the adaptive immune system.

\section{Immunomodulation by parasites}

This type of antigenic variation is not an option for the more long-lived helminth parasites, which survive as individual organisms for months or years. For these creatures, a more subtle but no less effective stratagem has 


\section{Protozoa \\ Unicellular, either intracellular (for example, malaria) or extracellular (for example, African trypanosomes). Malaria kills over 1 million per year.}

\section{Helminths}

Multicellular, metazoan worms; includes roundworms (nematodes), schistosomes and tapeworms.

Over $25 \%$ of global population infected.
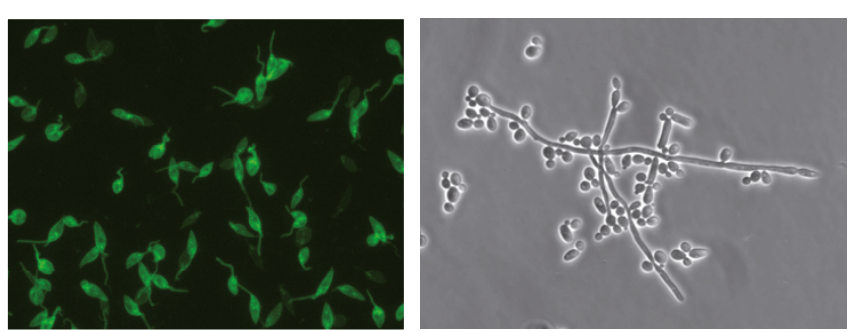

Fungi

Unicellular (yeast-like)

or multicellular

(hyphal); includes

common human

pathogens such as

Candida albicans.

Leishmania mexicana

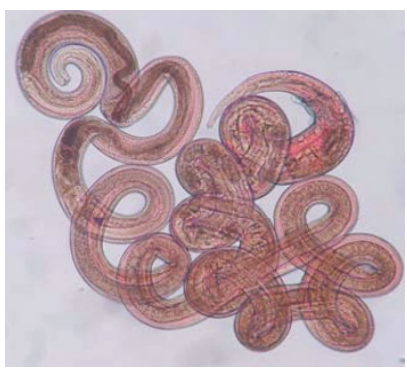

Heligmosomoides polygyrus
Candida albicans

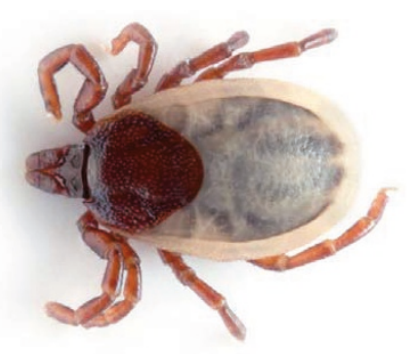

Ixodes hexagonus

\section{Ectoparasites}

Lice, mites, ticks and other arthropods.

Figure 1

Categories of parasites, using the broader definition to include fungi and ectoparasites. An ectoparasite (a louse of mice) has recently been found to exert similar immunomodulatory effects to those associated with gastrointestinal helminth infection in a wild wood mouse population [2]. Shown are, clockwise from top left, Leishmania mexicana protozoal promastigotes (L. Prieto-Lafuente); Candida albicans (from http:// www.dreamstime.com/stock-photo-of-candida-abicans-image894348); Ixodes hexagonus (from Wikipedia [15]); and Heligmosomoides polygyrus adult nematode worms (C Finney).

been to directly down-modulate the intensity and efficacy of host immune responses. It has been well established, for example, that patients carrying chronic schistosome or filarial infections lose the ability to mount parasitespecific $\mathrm{T}$ cell responses. This unresponsiveness has been attributed to parasite stimulation of endogenous host immunosuppressive controls both in humans [8] and animal models [9]. The best-understood of these suppressive systems is the regulatory $\mathrm{T}$ cell (Treg), a cellular safety catch on the immune response that normally blocks autoimmunity and reactivity to food antigens and allergens. Indeed, infection with the mouse gut nematode Heligmosomoides polygyrus stimulates Tregs that are able broadly to suppress responses to allergens [10].

In a recent study on 'real-world' infections, wild wood mice (Apodemus sylvaticus) infected with $H$. polygyrus were found to mount diminished cytokine responses following TLR stimulation [2], consistent with the broader immunosuppression seen in laboratory models. By targeting innate immune responses, the parasite is also reducing cytokine-based stimulation of the adaptive immune system. Moreover, similar effects were exerted by ectoparasites, in particular the louse Polyplax serrata. This is the first evidence of systemic down-regulation by arthropod parasites, raising the question of whether ectoparasite infestation could be an important environmental factor in human immune responsiveness. The need to resist down-regulation of TLR signaling by parasites may explain why some alleles of TLR4 and its co-receptor CD14 are associated with the development of allergic asthma [11].

\section{Polymorphism in the immune system}

Immune system genes are exceptionally polymorphic, reflecting in part selection by diverse and rapidly varying pathogens, but also the need to balance effective pathogen elimination against the risk of self-destructive reactions. This is well recognized for polymorphisms affecting the structural domains of proteins that function in pathogen recognition. It is less well recognized for the regulation of immune responses. The effect of parasites, for example, has been to dampen, rather than fully ablate, immune responsiveness, and the degree of immunosuppression varies markedly between pathogen species. These graduated effects may, in turn, have driven quantitative polymorphisms in the contemporary immune system that control the strength of the immune response, exemplified by nucleotide variation in regions controlling expression levels rather than variations in amino acid sequence in structural domains (Figure 2). 


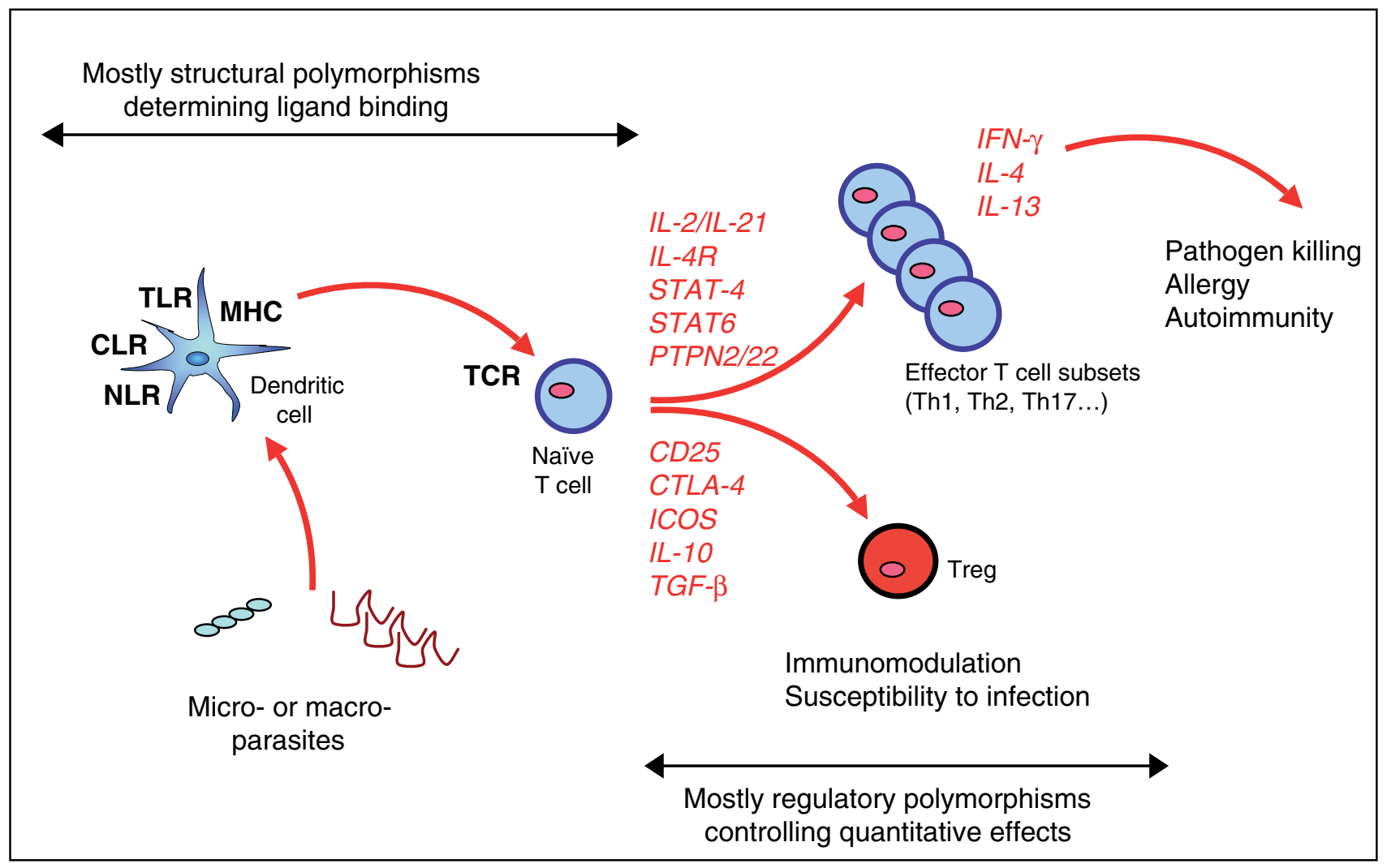

Figure 2

Schematic diagram of the polymorphic elements in immune responsiveness and where pathogen immunomodulation has driven evolution. In black bold type are the immune system families that have diversified primarily at the level of receptor-ligand specificities; those loci in red italic type are loci encoding cytokines, transcriptional regulators and cell surface molecules that are generally polymorphic in promoter, intronic and 3' UTR sequences suggesting a regulatory or quantitative effect of polymorphism. Loci above the bifurcation generally determine T cell activation, and those below down-regulation, although the distinction is blurred: for example, IL-2 promotes both effector T cell proliferation and Treg survival. CLR, C-type lectin receptors, which recognize conserved glycans of pathogens; NLR, NOD-like receptors, intracellular receptors that recognize pathogen products; TLR, Toll-like receptors, which recognize conserved molecular ligands from pathogens; MHC, major histocompatibility molecules, which bind peptide fragments of pathogen proteins and display them for recognition by T cells; TCR, T cell receptor, the highly variable receptor through which T cells recognize their targets.

This may be the explanation for the link between pathogen richness (number of diverse species) and host genetic diversity that has recently been documented in a report on cytokine gene polymorphism by Fumagalli et al. [3]. In an analysis of nearly 100 human interleukin genes, they found the highest single nucleotide polymorphism (SNP) frequencies in geographical areas with the highest number of endemic helminth species; those loci showing greatest variability included some encoding cytokines controlling both innate immune responses (such as the IL-1 family) and adaptive Th2 responses (such as IL-4 and IL-4R). Strikingly (in terms of the hygiene hypothesis) 6 out of 9 alleles known to predispose to inflammatory bowel disease (an immunopathology due to reactivity with commensal bacteria) were more frequent in pathogen-rich locations.
Earlier studies have linked noncoding polymorphisms in immune gene variants previously identified as asthma predisposition loci with resistance to parasitic helminths [12]. For example, the IL-13 promoter allele -1055T increases risk of asthma, but decreases schistosome egg load [13]; similarly, non-coding variants of the transcriptional regulator STAT-6, which is on the IL-4 pathway, are associated with higher asthma incidence and decreased susceptibility to the roundworm Ascaris [12]. Most SNPs associated with both helminth resistance and predisposition to allergy appear to be in non-coding regulatory regions (promoters, intronic regions or $3^{\prime}$ untranslated regions (UTRs)), although some structural allelisms are known (for example, in IL-4R [12]). This suggests that, in the main, parasite-maintained polymorphisms control the intensity of an immune 
response (or indeed, the strength of a suppressive Treg effect). Such 'allelic rheostats' are also known in autoimmunity-associated loci: for example, in one cohort of systemic lupus erythromatosus patients, the frequency of circulating Tregs was depressed in those carrying a disease-associated $3^{\prime}$ UTR SNP allele of CTLA-4, a surface molecule of $\mathrm{T}$ cells that acts as a brake on $\mathrm{T}$ cell activation [14].

\section{Allelic rheostats}

As with TLR polymorphisms, the presence of allelic forms for many adaptive immune system genes (in particular, at Treg-associated loci) suggests that there is no certain genetic optimum and that, in an environment with diverse pathogens demanding conflicting response patterns, the fine-tuning effect of multiple allelic variants allows the immune system to be variably calibrated across the population. In the absence of infection, and where genotypes tend to the higher end of reactivity (for example, where they result in low Treg frequencies), the immune response is more likely to overshoot, and responses may develop to innocuous targets such as self-antigens and allergens $[1,2]$.

\section{Keeping a balance}

Although the hygiene hypothesis is couched in very general terms, there is strong evidence that specific geneenvironment (and more specifically, gene-parasite) interactions can contribute to the development of damaging immune reactions in autoimmunity and allergy. The identification of precise genetic variants controlling both parasite susceptibility and immunopathology offers the possibility of pinpointing mechanisms that require inhibition or amplification for treatment of disease, identifying genotypes that may be exceptionally susceptible to either infection or pathology, and a deeper understanding of the intimate co-evolution of pathogens and the immune system.

\section{Acknowledgements}

The author's research is supported by grants from the Wellcome Trust, the Medical Research Council and the European Commission contract INCO-CT-2006-032436.

\section{References}

1. Maizels RM: Infections and allergy - helminths, hygiene and host immune regulation. Curr Opin Immunol 2005, 17:656-661.
2. Jackson JA, Friberg IM, Bolch L, Lowe A, Ralli C, Harris PD, Behnke JM, Bradley JE: Immunomodulatory parasites and toll-like receptor-mediated tumour necrosis factor alpha responsiveness in wild mammals. BMC Biology 2009, 7:16.

3. Fumagalli M, Pozzoli U, Cagliani R, Comi GP, Riva S, Clerici M, Bresolin N, Sironi M: Parasites represent a major selective force for interleukin genes and shape the genetic predisposition to autoimmune conditions. J Exp Med 2009, 206:1395-1408.

4. Miller SI, Ernst RK, Bader MW: LPS, TLR4 and infectious disease diversity. Nat Rev Microbiol 2005, 3:36-46.

5. Roach JC, Glusman G, Rowen L, Kaur A, Purcell MK, Smith KD, Hood LE, Aderem A: The evolution of vertebrate Tolllike receptors. Proc Natl Acad Sci USA 2005, 102:9577-9582.

6. Sun J, Walsh M, Villarino AV, Cervi L, Hunter CA, Choi Y, Pearce EJ: TLR ligands can activate dendritic cells to provide a MyD88-dependent negative signal for Th2 cell development. J Immunol 2005, 174:742-751.

7. Schmid-Hempel P: Parasite immune evasion: a momentous molecular war. Trends Ecol Evol 2008, 23:318-326.

8. Babu S, Blauvelt CP, Kumaraswami V, Nutman TB: Regulatory networks induced by live parasites impair both Th1 and Th2 pathways in patent lymphatic filariasis: implications for parasite persistence. J Immunol 2006, 176:3248-3256.

9. Taylor M, Le Goff L, Harris A, Malone E, Allen JE, Maizels RM: Removal of regulatory $\mathrm{T}$ cell activity reverses hyporesponsiveness and leads to filarial parasite clearance in vivo. J Immunol 2005, 174:4924-4933.

10. Wilson MS, Taylor M, Balic A, Finney CAM, Lamb JR, Maizels RM: Suppression of allergic airway inflammation by helminth-induced regulatory $\mathbf{T}$ cells. J Exp Med 2005, 202:1199-1212.

11. Smit LAM, Siroux V, Bouzigon E, Oryszczyn M-P, Lathrop M, Demenais F, Kauffmann F: CD14 and toll-like receptor gene polymorphisms, country living, and asthma in adults. $\mathrm{Am} J$ Respir Crit Care Med 2009, 179:363-368.

12. Moller M, Gravenor MB, Roberts SE, Sun D, Gao P, Hopkin $\mathrm{JM}$ : Genetic haplotypes of Th-2 immune signalling link allergy to enhanced protection to parasitic worms. Hum Mol Genet 2007, 16:1828-1836.

13. Kouriba B, Chevillard C, Bream JH, Argiro L, Dessein $H$, Arnaud V, Sangare L, Dabo A, Beavogui AH, Arama C, Traoré HA, Doumbo O, Dessein A: Analysis of the $5 q 31-q 33$ locus shows an association between IL13-1055C/T IL-13-591A/G polymorphisms and Schistosoma haematobium infections. J Immunol 2005, 174:6274-6281.

14. Barreto M, Ferreira RC, Lourenço L, Moraes-Fontes MF, Santos E, Alves M, Carvalho C, Martins B, Andreia R, Viana JF, Vasconcelos C, Mota-Vieira L, Ferreira C, Demengeot J, Vicente AM: Low frequency of $\mathrm{CD4}^{+} \mathrm{CD25} 5^{+}$Treg in SLE patients: a heritable trait associated with CTLA4 and TGFb gene variants. BMC Immunol 2009, 10:5.

15. Wikipedia [http://en.wikipedia.org/wiki/File:Tick_2_(aka).jpg]

Published: 5 August 2009

doi:10.1186/jbiol166

(c) 2009 BioMed Central Ltd 